\title{
Green Synthesis of Silver Nanoparticles with Antibacterial Activities Using Aqueous Eriobotrya Japonica Seed Extract
}

\author{
Bo RAO ${ }^{a}$ and Ren-Cheng TANG ${ }^{b^{*}}$ \\ National Engineering Laboratory for Modern Silk, College of Textile and Clothing Engineering, \\ Soochow University, 199 Renai Road, Suzhou 215123, China \\ a 535184812@qq.com.cn, ${ }^{\text {b* }}$ tangrencheng@suda.edu.cn
}

Keywords: Antibacterial activity, Eriobotrya japonica, Green synthesis, Silver nanoparticles.

\begin{abstract}
An eco-friendly approach for the preparation of silver nanoparticles (AgNPs) from silver nitrate solution using aqueous Eriobotrya japonica seed extract was investigated. The reduction of silver ions in solution was monitored using UV-vis spectroscopy, and the surface plasmon resonance of AgNPs at $428 \mathrm{~nm}$ was observed. The biosynthesized nanoparticles were characterized using transmission electron microscopy (TEM), scanning electron microscopy, energy dispersive X-ray spectroscopy, dynamic light scattering and X-ray diffraction. The prepared AgNPs were polydispersed and spherical in shape, and their average particle size determined by TEM was $25 \mathrm{~nm}$. Furthermore the biosynthesized AgNPs were found to exhibit effective antibacterial activities against Escherichia coli and Staphylococcus aureus.
\end{abstract}

\section{Introduction}

In the past ten years, silver nanoparticles (AgNPs) have attracted a great deal of attention due to their unique physical, chemical, optical and biological properties, and found tremendous applications in various industries such as biomedicine, drug delivery, electronics, optics, catalysis, food industry, textile industry, etc. [1]. A variety of chemical and physical approaches have been developed to prepare AgNPs. Among them, the chemical reduction is most widely used. However, these approaches are inevitably associated with the use of hazardous chemicals such as reductants, stabilizers and organic solvents, or have special requirements for the employed techniques such as high energy radiation, microwave irradiation, etc. [2, 3]. In recent years, the biological approach using plant extracts has become a valuable alternative to physical and chemical synthesis. Many researches have been reported on the biosynthesis of AgNPs plant extracts [4].

Eriobotrya japonica (loquat) is a large evergreen shrub or small tree and cultivated wordwide in all warm and subtropical regions for its fruit or as an ornamental plant. But up to now the seeds of $E$. japonica have usually been disposed of as a waste after consuming its fruits. In terms of the comprehensive utilization of E. japonica seeds as well as the recent developments of plant-mediated AgNPs synthesis, it is significant to carry out the research on the biosynthesis of AgNPs using the extracts of E. japonica seeds. The aqueous E. japonica seed extract is rich in nutritional and bioactive components such as amygdalins, polyphenols, proteins, etc. [5], which could be used as reductants for the synthesis of AgNPs from silver nitrate solution and also stabilizers of generated AgNPs. In this study, a green approach for the synthesis of AgNPs using aqueous E. japonica seed extract was described. And the AgNPs obtained were afterwards subjected to a sequence of characterizations. In addition, the antibacterial activities of AgNPs against Escherichia coli and Staphylococcus aureus were examined.

\section{Experimental}

Materials. Silver nitrate was purchased from Sinopharm Chemical Reagent Co. Ltd., China. Eriobotrya japonia seed was collected from the orchard in the suburb of Suzhou, China. 
Preparation of $\boldsymbol{E}$. Japonia Seed Extract. To prepare the aqueous E. japonia seed extract, the mixture containing $5 \mathrm{~g}$ of the seed powder and $100 \mathrm{~mL}$ of deionized water in the $250 \mathrm{~mL}$ Erlenmeyer flask was heated in the oscillator at $90{ }^{\circ} \mathrm{C}$ for $60 \mathrm{~min}$. Subsequently, the extract was centrifuged at $4000 \mathrm{rpm}$ for $20 \mathrm{~min}$ to remove impurities, The supernatant was collected, diluted by deionized water to $100 \mathrm{~mL}$, and finally stored at $-4{ }^{\circ} \mathrm{C}$ for further experiments.

Biosynthesis of AgNPs. For the typical reduction of silver ions to AgNPs, $5 \mathrm{~mL}$ of the freshly prepared extract was added to $50 \mathrm{~mL}$ of the aqueous silver nitrate solution $(1 \mathrm{mM})$, and then the $\mathrm{pH}$ value of the mixture was immediately adjusted to 8 using $0.1 \mathrm{M}$ sodium hydroxide before incubation at $60{ }^{\circ} \mathrm{C}$. As the reaction proceeded, the color change of the solution from colorless to yellowish brown was observed and recognized for the formation of AgNPs.

Measurements. The UV-vis spectra of the mixture before and after reaction were measured by UV-1800 UV-vis spectrophotometer from 200 to $800 \mathrm{~nm}$. The particle size distribution of the prepared AgNPs colloid was determined by Marlvern Zetasizer Nano ZS 90 at a scattering angle of $90^{\circ}$. The detailed size and morphology of biosynthesized AgNPs was investigated by using S-4800 scanning electron microscope with energy dispersive spectrometer and HT700 transmission electron microscope. The X-ray diffraction measurement of AgNPs was performed on the X'Pert-Pro MPD $\mathrm{X}$-ray diffractometer equipped using the $\mathrm{Cu}-\mathrm{K} \alpha$ radiation of wavelength $0.15418 \mathrm{~nm}$. The antibacterial activity of biosynthesized AgNPs against $E$. coli and S. aureus was determined using the well diffusion method.

\section{Results and Discussion}

UV-vis Spectral Studies. Fig. 1a displays the color change of the mixture before and after reaction. The formation of AgNPs in the solution was evidenced by the color change from colorless to yellowish brown owing to the excitation of the surface plasmon resonance of AgNPs [6]. Fig. 1b shows the UV-vis absorption spectra of the mixture of E. japonia seed extract and silver nitrate as a function of time. The bioreduction of silver ions gave rise to the appearance of the characteristic absorption peak at around $428 \mathrm{~nm}$. The peak intensity increased with an increase in reaction time, indicating the increasing number of AgNPs formed in the mixture. The UV-vis spectrum recorded after 90 min showed no obvious increase, suggesting the reaction tended towards equilibrium.
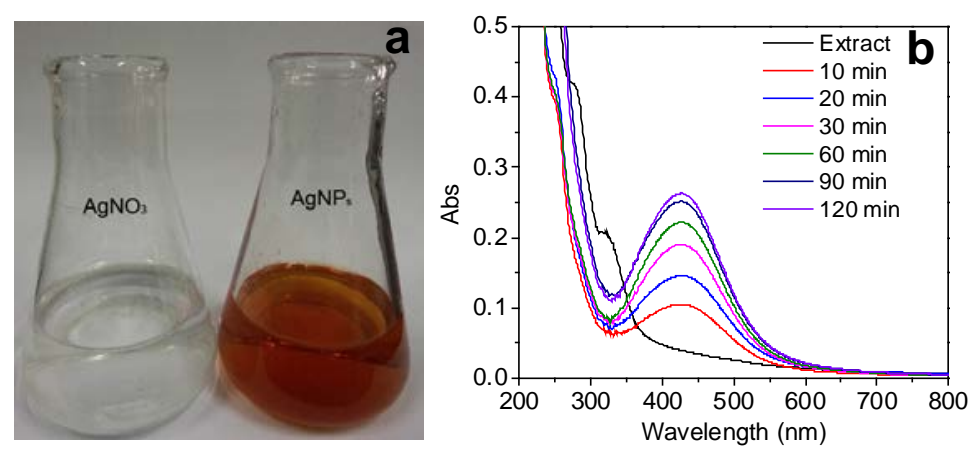

Fig. 1. Color change of silver nitrate solution before and after the synthesis of AgNPs for 60 min (a) and UV-vis absorption spectra of the reaction mixture of E. japonia seed extract and $\mathrm{AgNO}_{3}$ as a function of time (b).

Characterizations of Biosynthesized AgNPs. The SEM, EDX, TEM and DLS techniques were applied to determine the size, shape and morphologies of AgNPs in order to give further insights into the detailed features of AgNPs. The SEM image obtained at a high magnification is shown in Fig. 2a, and the related EDX spectrum is given in Fig. 2b. The AgNPs obtained were polydispersed and spherical in shape with a size distribution in the range from 14 to $76 \mathrm{~nm}$. A small number of large 
particles viewed probably resulted from the agglomeration of small ones. The EDX profile showed strong signal peak at $3 \mathrm{keV}$, which is typical of the absorption of silver nanocrystallites [7], demonstrating the presence of AgNPs. Interestingly, some adjoint elements such as $\mathrm{C}, \mathrm{O}$ and $\mathrm{Cl}$ were also detected. This is very likely associated with the organic moieties from the extract adsorbed on the surface of AgNPs, which play a crucial role in the reduction and stabilization of AgNPs.

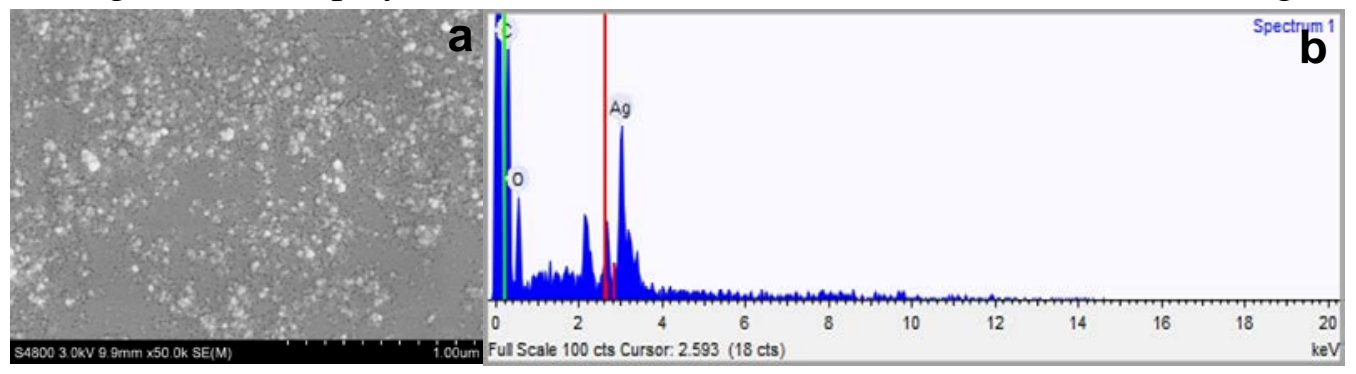

Fig. 2. SEM image of biosynthesized AgNPs using E. japonia seed extract (a) and EDX spectrum of biosynthesized AgNPs (b).

The morphology and size dimension of the biosynthesized AgNPs can be intuitively obtained from the TEM image ( Fig. 3a) and the size distribution in the colloid can be given by the DLS analysis (Fig. 3c). The AgNPs were spherical in shape with different size distribution. It should be noted that the average particle size (around $25 \mathrm{~nm}$ ) shown by Fig. 3b obtained from TEM analysis was significantly smaller than that (about $100 \mathrm{~nm}$ ) measured by the DLS method. This discrepancy could be possibly due to the adsorption of organic stabilizers from the extract on the surface of AgNPs, the aggregation of some small particles and the adsorption of water on the stabilized AgNPs [8,9], all of which could have a negative effect on the average particle size obtained by the DLS method.
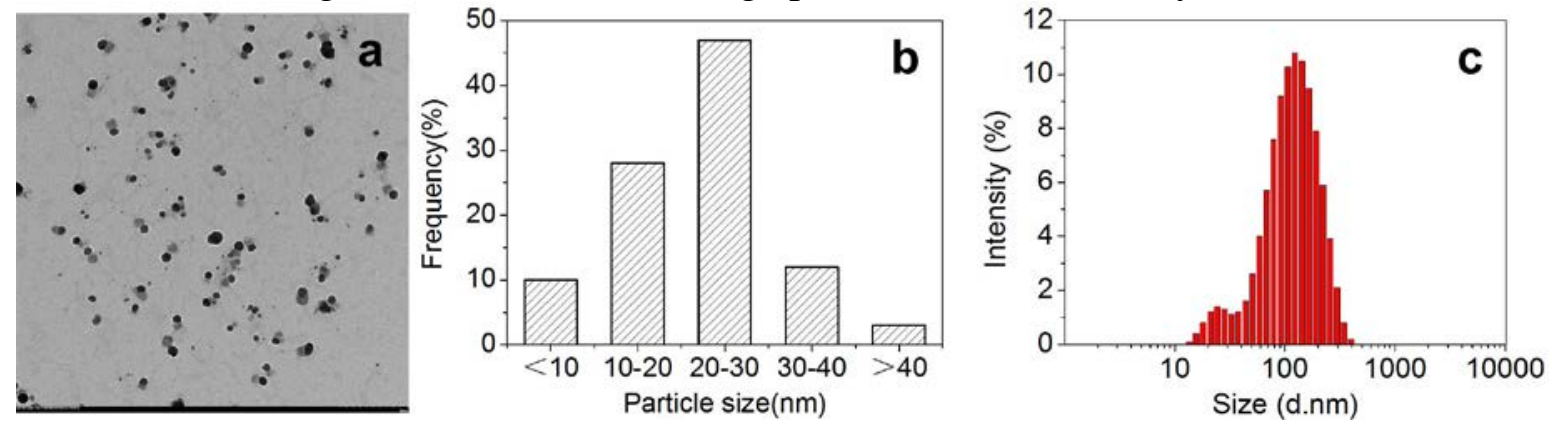

Fig. 3. TEM image of biosynthesized AgNPs using E. japonica seed extract (a), histogram of the AgNPs size distribution (b) and DLS histogram of biosynthesized AgNPs in colloidal solution (c).

Fig. 4 illustrates the XRD pattern of the biosynthesized AgNPs using E. japonica seed extract, which can confirm the crystalline nature of the prepared AgNPs. The diffraction peaks at the $2 \theta$ values of $32.22^{\circ}, 38.17^{\circ}, 46.26^{\circ}, 54.91^{\circ}, 57.79^{\circ}$ and $77.14^{\circ}$ corresponded to the $122,123,231,142$, 241 and 311 crystallographic planes, respectively. This suggests that the prepared AgNPs are biphasic in nature. And the similar crystalline structure obtained by XRD was also found in the biosynthesized AgNPs using Ocimum canum leaf extract [10].

Antibacterial Activity. As shown by Fig. 5, the zone of inhibition was clearly observed in the vicinity of wells filled with silver nitrate (9.3 mm for E. coli, and $7.5 \mathrm{~mm}$ for S. arueus), and AgNPs ( $8.5 \mathrm{~mm}$ for E. coli, and $7.3 \mathrm{~mm}$ for S. arueus). The E. japonica seed extract did not display the obvious zone of inhibition. The tests prove that both silver nitrate and biosynthesized AgNPs have good antibacterial activities against E. coli and S. aureus. 


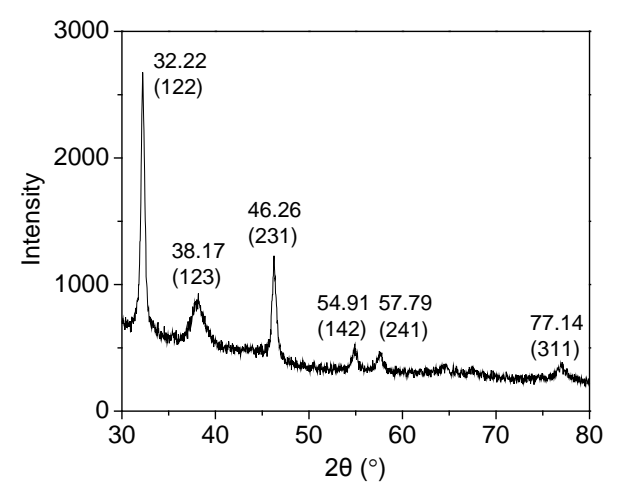

Fig. 4. XRD pattern of biosynthesized AgNPs using E. japonica seed extract.
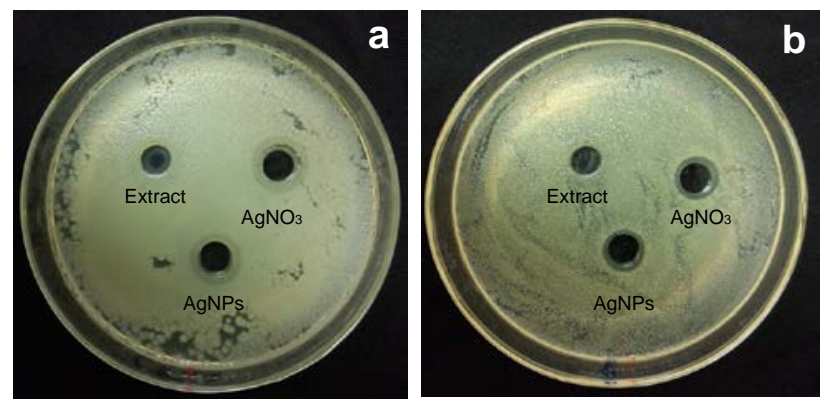

Fig. 5. Antibacterial activities of biosynthesized AgNPs using E. japonica seed extract against E. coli (a) and S. aureus (b).

\section{Conclusions}

An eco-friendly and cost-effective protocol for the synthesis of AgNPs by utilizing a renewable natural resource E. japonica seed was proposed. The biosynthesized AgNPs were characterized by UV-vis, SEM, EDX, TEM, DLS and XRD. The AgNPs were polydispersed in solution and spherical in shape, and their average particle sizes determined by TEM and DLS were about 25 and $100 \mathrm{~nm}$, respectively. The bioge nic AgNPs exhibited good antibacterial activities against $E$. coli and $S$. aureus. Further research on the AgNPs biosynthesized using E. japonica seed extract could bring a promising application in the fields of medicine and hygiene.

\section{Acknowledgement}

This study was funded by Jiangsu Provincial Natural Science Foundation of China (BK20131178), and Jiangsu Provincial Key Research and Development Program of China (BE2015066).

\section{References}

[1] V.K. Sharma, R.A. Yngard, Y. Lin, Adv. Colloid Interfac. Sci. 145 (2008) 83-86.

[2] H.S. Shin, H.J. Yang, S.B. Kim, M.S. Lee, J. Colloid Interf. Sci. 274 (2004) 89-94.

[3] M. Noroozi, A. Zakaria, M.M. Moksin, Z.A. Wahab, A. Abedini, Int. J. Mol. Sci. 13 (2012) 8086-8096.

[4] M.S. Akhtar, J. Panwar, Y.-S. Yun, ACS Sustain. Chem. Eng. 1 (2013) 591-602.

[5] E.-N. Li, J.-G. Luo, L.-Y. Kong, Phytochem. Anal. 20 (2009) 338-343.

[6] A. Henglein, J. Phys. Chem. 97 (1993) 5457-5471. 
[7] Y. Liu, M. Hussain, H. Memon, S. Yasin, Dig. J. Nanomater. Bios. 10 (2015) 1019-1024.

[8] A. Bootz, V. Vogel, D. Schubert, J. Kreuter, Eur. J. Pharm. Biopharm. 57 (2004) 369-375.

[9] S. Das, P. Roy, S. Mondal, T. Bera, A. Mukherjee, Colloid. Surface. B 107 (2013) 27-34.

[10] C. Jayaseelan, A.A. Rahuman, Parasitol Res. 111 (2012) 1369-1378. 